\title{
On the Period Length of Pseudorandom Vector Sequences Generated by Matrix Generators
}

\author{
By Jürgen Eichenauer-Herrmann, Holger Grothe, and Jürgen Lehn
}

\begin{abstract}
In Tahmi [5], Niederreiter [4], Afflerbach and Grothe [1], and Grothe [2] linear recursive congruential matrix generators for generating $r$-dimensional pseudorandom vectors are analyzed. In particular, conditions are established which ensure that the period length equals $p^{r}-1$ for any nonzero starting vector in case of a prime modulus $p$. For a modulus of the form $p^{\alpha}, \alpha \geq 2$ and $p$ prime, this paper describes a simple method for constructing matrix generators having the maximal possible period length $\left(p^{r}-1\right) \cdot p^{\alpha-1}$ for any starting vector which is nonzero modulo $p$.
\end{abstract}

1. Introduction and Notation. A linear recursive congruential matrix generator for generating $r$-dimensional pseudorandom vectors is of the form

$$
\vec{x}_{n+1} \equiv A \cdot \vec{x}_{n}(\bmod m), \quad \vec{x}_{n+1} \in \mathbf{Z}_{m}^{r}, n \geq 0,
$$

where the modulus $m$ is a positive integer, $\mathbf{Z}_{m}=\{0,1, \ldots, m-1\}, \vec{x}_{0} \in \mathbf{Z}_{m}^{r}$, and $A \in \mathbf{Z}_{m}^{r \times r}$, i.e., $A$ is an $r \times r$-matrix with elements in $\mathbf{Z}_{m}$. In the sequel it is assumed that the matrix $A$ is nonsingular modulo $m$. Then the vector sequence $\left(\vec{x}_{n}\right)_{n \geq 0}$ generated by (1) is purely periodic, and the smallest positive integer $\lambda=$ $\lambda\left(A, \vec{x}_{0}, m\right)$ with $\vec{x}_{\lambda}=\vec{x}_{0}$ is called the period length of the vector sequence $\left(\vec{x}_{n}\right)_{n \geq 0}$. Analogously, the matrix sequence $\left(A_{n}\right)_{n \geq 0}$ with $A_{n} \equiv A^{n}(\bmod m), A_{n} \in \mathbf{Z}_{m}^{r \times r}$, is purely periodic, and the smallest positive integer $\lambda=\lambda(A, m)$ for which $A_{\lambda}$ equals the identity matrix $I$ is called the period length of the matrix sequence $\left(A_{n}\right)_{n \geq 0}$. The following two remarks are immediate consequences of these definitions.

Remark 1 . The period length $\lambda\left(A, \vec{x}_{0}, m\right)$ of the vector sequence $\left(\vec{x}_{n}\right)_{n \geq 0}$ divides the period length $\lambda(A, m)$ of the matrix sequence $\left(A_{n}\right)_{n \geq 0}$ for any starting vector $\vec{x}_{0} \in \mathbf{Z}_{m}^{r}$.

Remark 2. If $A_{\nu}=I$ for some positive integer $\nu$, then the period length $\lambda(A, m)$ of the matrix sequence $\left(A_{n}\right)_{n \geq 0}$ divides $\nu$.

It is well known (cf. Tahmi [5], Niederreiter [4], and Grothe [2]) that $\lambda\left(A, \vec{x}_{0}, p\right)=$ $\lambda(A, p)=p^{r}-1$ for any starting vector $\vec{x}_{0} \in \mathbf{Z}_{p}^{r} \backslash\{\overrightarrow{0}\}$ in case of a prime modulus $m=p$ if the characteristic polynomial of the matrix $A$ is primitive modulo $p$. In this paper the case of a modulus $m=p^{\alpha}, \alpha \geq 2$, is considered where $p$ is a prime number. It is shown that for $p \geq 3$ or $r \geq 2$ there exist matrix generators (1) with period length $\left(p^{r}-1\right) \cdot p^{\alpha-1}$ for any starting vector which is nonzero modulo $p$, and a simple method is described for determining such a generator. Observe that $\left(p^{r}-1\right) \cdot p^{\alpha-1}$ is the maximal possible period length according to the following technical lemma.

Received March 1, 1988.

1980 Mathematics Subject Classification (1985 Revision). Primary 65C10; Secondary 11K45.

Key words and phrases. Pseudorandom vector sequences, matrix generator, period length. 


\section{Matrix Generators with Maximal Period Length.}

LEMMA. Let $A \in \mathbf{Z}_{p^{\alpha+1}}^{r \times r}, \alpha \geq 1$, be a matrix which is nonsingular modulo $p$, and define matrices $A_{n} \in \mathbf{Z}_{p^{\alpha+1}}^{r \times r}$ by $A_{n} \equiv A^{n}\left(\bmod p^{\alpha+1}\right), n \geq 0$. Let $\lambda_{\alpha}=\lambda\left(A, p^{\alpha}\right)$ and $\lambda_{\alpha+1}=\lambda\left(A, p^{\alpha+1}\right)$ denote the period lengths of the matrix sequence $\left(A_{n}\right)_{n \geq 0}$ modulo $p^{\alpha}$ and modulo $p^{\alpha+1}$, respectively. Then

$$
\lambda_{\alpha+1}= \begin{cases}\lambda_{\alpha} & \text { for } A^{\lambda_{\alpha}} \equiv I\left(\bmod p^{\alpha+1}\right) \\ \lambda_{\alpha} \cdot p & \text { for } A^{\lambda_{\alpha}} \not \equiv I\left(\bmod p^{\alpha+1}\right)\end{cases}
$$

Proof. From $A^{\lambda_{\alpha}} \equiv I\left(\bmod p^{\alpha}\right)$ it follows that $A^{\lambda_{\alpha}}=I+p^{\alpha} \cdot B$ for some matrix $B \in \mathbf{Z}^{r \times r}$. Therefore,

$$
A^{\lambda_{\alpha} \cdot p}=\left(I+p^{\alpha} \cdot B\right)^{p}=I+\left(\begin{array}{c}
p \\
1
\end{array}\right) \cdot p^{\alpha} \cdot B+\left(\begin{array}{l}
p \\
2
\end{array}\right) \cdot\left(p^{\alpha} \cdot B\right)^{2}+\cdots+\left(p^{\alpha} \cdot B\right)^{p},
$$

which yields $A^{\lambda_{\alpha} \cdot p} \equiv I\left(\bmod p^{\alpha+1}\right)$, i.e., $\lambda_{\alpha+1}$ divides $\lambda_{\alpha} \cdot p$ according to Remark 2 . From $A^{\lambda_{\alpha+1}} \equiv I\left(\bmod p^{\alpha+1}\right)$ it follows that $A^{\lambda_{\alpha+1}} \equiv I\left(\bmod p^{\alpha}\right)$, i.e., $\lambda_{\alpha}$ divides $\lambda_{\alpha+1}$ according to Remark 2, which proves the lemma.

The purpose of this paper is to prove the following result.

THEOREM. Let $B \in \mathbf{Z}_{p^{\alpha}}^{r \times r}, \alpha \geq 2$, be a matrix whose characteristic polynomial is primitive modulo $p$. Then

$$
B^{p^{r}-1} \equiv I+p \cdot C\left(\bmod p^{2}\right)
$$

for some matrix $C \in \mathbf{Z}_{p}^{r \times r}$. Let $D \in \mathbf{Z}_{p^{\alpha-1}}^{r \times r}$ denote an arbitrary matrix with $B \cdot D \equiv$ $D \cdot B(\bmod p)$,

$$
\operatorname{det}(D) \not \equiv 0(\bmod p) \quad \text { for } p \geq 3
$$

and

$$
\operatorname{det}(D) \equiv \operatorname{det}(D+I) \equiv 1(\bmod 2) \quad \text { for } p=2 .
$$

Define a matrix $A \in \mathbf{Z}_{p^{\alpha}}^{r \times r}$ by

$$
A \equiv B \cdot(I+p \cdot(C-D))\left(\bmod p^{\alpha}\right) .
$$

Then the period length of the vector sequence $\left(\vec{x}_{n}\right)_{n \geq 0}$ generated according to (1) with matrix $A$ and modulus $m=p^{\alpha}$ is given by

$$
\lambda\left(A, \vec{x}_{0}, p^{\alpha}\right)=\left(p^{r}-1\right) \cdot p^{\alpha-1}
$$

for any starting vector $\vec{x}_{0} \in \mathbf{Z}_{p^{\alpha}}^{r}$ with $\vec{x}_{0} \not \equiv \overrightarrow{0}(\bmod p)$.

Proof. The proof is subdivided into four parts (i) to (iv).

(i) Because of $A \equiv B(\bmod p)$ according to (5) it follows that

$$
\lambda\left(A, \vec{x}_{0}, p\right)=\lambda(A, p)=p^{r}-1
$$

for any starting vector $\vec{x}_{0} \in \mathbf{Z}_{p}^{r} \backslash\{\overrightarrow{0}\}$, since the characteristic polynomial of the matrix $B$ is primitive modulo $p$. In particular, $B^{p^{r}-1} \equiv I(\bmod p)$ holds. Hence a matrix $C$ with (2) exists. Observe that $(2)$ yields $B \cdot C \equiv C \cdot B(\bmod p)$, which implies that $B \cdot(C-D) \equiv(C-D) \cdot B(\bmod p)$ because of the hypothesis $B \cdot D \equiv D \cdot B$ $(\bmod p)$. Therefore $(5)$ and $(2)$ yield

$$
\begin{aligned}
A^{p^{r}-1} & \equiv[B \cdot(I+p \cdot(C-D))]^{p^{r}-1} \equiv B^{p^{r}-1} \cdot\left(I+\left(p^{r}-1\right) \cdot p \cdot(C-D)\right) \\
& \equiv(I+p \cdot C) \cdot(I-p \cdot(C-D)) \equiv I+p \cdot D\left(\bmod p^{2}\right) .
\end{aligned}
$$


If $p=2$ then it follows from (7) that

$$
A^{2^{r}-1}=I+2 \cdot D+4 \cdot E
$$

for some matrix $E \in \mathbf{Z}^{r \times r}$ and hence

$$
A^{\left(2^{r}-1\right) \cdot 2}=(I+2 \cdot D+4 \cdot E)^{2}=I+4 \cdot D+4 \cdot D^{2}+8 \cdot F
$$

for some matrix $F \in \mathbf{Z}^{r \times r}$, i.e.,

$$
A^{\left(2^{r}-1\right) \cdot 2} \equiv I+4 \cdot D \cdot(D+I)(\bmod 8) .
$$

(ii) Now it is shown by induction that in case of $p \geq 3$,

$$
A^{\left(p^{r}-1\right) \cdot p^{\nu}} \equiv I+p^{\nu+1} \cdot D\left(\bmod p^{\nu+2}\right)
$$

for $0 \leq \nu \leq \alpha-2$. Obviously, (7) is equivalent to (8) for $\nu=0$. If (8) is valid for some $\nu$ with $0 \leq \nu \leq \alpha-3$, then

$$
A^{\left(p^{r}-1\right) \cdot p^{\nu}}=I+p^{\nu+1} \cdot D+p^{\nu+2} \cdot E_{\nu}
$$

for some matrix $E_{\nu} \in \mathbf{Z}^{r \times r}$ and hence

$$
A^{\left(p^{r}-1\right) \cdot p^{\nu+1}}=\left(I+p^{\nu+1} \cdot\left(D+p \cdot E_{\nu}\right)\right)^{p}=I+p^{\nu+2} \cdot\left(D+p \cdot E_{\nu}\right)+p^{\nu+3} \cdot F_{\nu}
$$

for some matrix $F_{\nu} \in \mathbf{Z}^{r \times r}$ because of $p \geq 3$, which yields

$$
A^{\left(p^{r}-1\right) \cdot p^{\nu+1}} \equiv I+p^{\nu+2} \cdot D\left(\bmod p^{\nu+3}\right)
$$

Therefore (8) holds for $0 \leq \nu \leq \alpha-2$. It can be similarly proved that in case of $p=2$,

$$
A^{\left(2^{r}-1\right) \cdot 2^{\nu}} \equiv I+2^{\nu+1} \cdot D \cdot(D+I)\left(\bmod 2^{\nu+2}\right)
$$

for $1 \leq \nu \leq \alpha-2$.

(iii) Because of $(3),(4),(6),(7),(8)$ and (9) it follows from the lemma that

$$
\lambda\left(A, p^{\nu+1}\right)=\left(p^{r}-1\right) \cdot p^{\nu}
$$

for $0 \leq \nu \leq \alpha-1$. Note that if $\vec{x}_{0} \not \equiv \overrightarrow{0}(\bmod p)$, then

$$
D \cdot \vec{x}_{0} \not \equiv \overrightarrow{0}(\bmod p) \quad \text { for } p \geq 3
$$

and

$$
D \cdot(D+I) \cdot \vec{x}_{0} \not \equiv \overrightarrow{0}(\bmod p) \quad \text { for } p=2
$$

because of (3) and (4), respectively. Therefore (7), (8) and (9) show that

$$
A^{\left(p^{r}-1\right) \cdot p^{\nu}} \cdot \vec{x}_{0} \not \equiv \vec{x}_{0}\left(\bmod p^{\nu+2}\right)
$$

for $\vec{x}_{0} \not \equiv \overrightarrow{0}(\bmod p)$ and $0 \leq \nu \leq \alpha-2$.

(iv) Now it is proved by induction that

$$
\lambda\left(A, \vec{x}_{0}, p^{\nu+1}\right)=\left(p^{r}-1\right) \cdot p^{\nu}
$$

for any starting vector $\vec{x}_{0} \in \mathbf{Z}_{p^{\alpha}}^{r}$ with $\vec{x}_{0} \not \equiv \overrightarrow{0}(\bmod p)$ and $0 \leq \nu \leq \alpha-1$. Obviously, (6) is equivalent to (12) for $\nu=0$. Now assume that (12) is valid for some $\nu$. with $0 \leq \nu \leq \alpha-2$. Then

$$
\lambda\left(A, \vec{x}_{0}, p^{\nu+2}\right)=\mu \cdot\left(p^{r}-1\right) \cdot p^{\nu}
$$


for some integer $\mu \geq 1$. Since

$$
\lambda\left(A, \vec{x}_{0}, p^{\nu+2}\right) \neq\left(p^{r}-1\right) \cdot p^{\nu}
$$

according to (11), it follows that $\mu>1$. Remark 1 and (10) imply that $\lambda\left(A, \vec{x}_{0}, p^{\nu+2}\right)$ divides $\left(p^{r}-1\right) \cdot p^{\nu+1}$ and hence $\mu=p$, which proves the theorem.

Observe that there exist primitive polynomials of degree $r$ over the Galois field $\mathrm{GF}(p)$ for every positive integer $r$ and every prime number $p$. Such a polynomial, and hence a matrix $B \in \mathbf{Z}_{p^{\alpha}}^{r \times r}$ which satisfies the hypothesis of the theorem, can be determined without any effort if $p$ and $r$ are small integers (see, e.g., Knuth $[3$, p. 28]).

Since the characteristic polynomial of the matrix $B$ is primitive modulo $p$, it follows that $\operatorname{det}(B) \not \equiv 0(\bmod p)$ and that $B \cdot \vec{x}_{0} \not \equiv \vec{x}_{0}(\bmod 2)$ for $p=2, r \geq 2$, and $\vec{x}_{0} \not \equiv \overrightarrow{0}(\bmod 2)$. Hence $\operatorname{det}(B+I) \equiv 1(\bmod 2)$ for $p=2$ and $r \geq 2$. Therefore, the matrix $D \in \mathbf{Z}_{p^{\alpha-1}}^{r \times r}$ with $D \equiv B\left(\bmod p^{\alpha-1}\right)$ satisfies the hypothesis of the theorem if $p \geq 3$ or $r \geq 2$.

Acknowledgment. The authors are indebted to Professor H. Niederreiter for valuable hints given in a discussion on the topic of this paper. They also would like to thank the Deutsche Forschungsgemeinschaft for financial support.

Technische Hochschule Darmstadt

Fachbereich Mathematik

SchloBgartenstr. 7

D-6100 Darmstadt, West Germany

E-mail: x\%xbr1dc3i@ddathd21.bitnet

1. L. AfFLERBACH \& H. GROThe, "The lattice structure of pseudo-random vectors generated by matrix generators," J. Comput. Appl. Math., v. 23, 1988, pp. 127-131.

2. H. GROTHE, "Matrix generators for pseudo-random vector generation," Statist. Hefte, v. 28, (1987), pp. 233-238.

3. D. E. KNUTh, The Art of Computer Programming, Vol 2, 2nd ed., Addison-Wesley, Reading, Mass, 1981.

4. H. NiEderReiter, "A pseudorandom vector generator based on finite field arithmetic," Math. Japon., v. 31, 1986, pp. 759-774.

5. E.-H. A. D. E. TAнMI, Contribution aux Générateurs de Vecteurs Pseudo-Aléatoires, Thèse, Université des Sciences et de la Technologie Houari Boumedienne, Algier, 1982. 